\title{
Technè
}

La science au service de l'histoire de l'art et de la préservation des biens culturels

$40 \mid 2014$

Thérapéia. Polychromie et restauration de la sculpture dans l'Antiquité

\section{Vie et mort des offrandes à Délos}

The life and death of offerings in Delos

\section{Clarisse Prêtre}

\section{(2) OpenEdition}

1 Journals

\section{Édition électronique}

URL : https://journals.openedition.org/techne/3307

DOI : 10.4000/techne.3307

ISSN : 2534-5168

Éditeur

C2RMF

\section{Édition imprimée}

Date de publication : 26 novembre 2014

Pagination : $35-41$

ISBN : 978-2-7118-6218-4

ISSN : $1254-7867$

\section{Référence électronique}

Clarisse Prêtre, «Vie et mort des offrandes à Délos », Technè [En ligne], 40 | 2014, mis en ligne le 24 juillet 2020, consulté le 08 février 2022. URL : http://journals.openedition.org/techne/3307 ; DOI : https://doi.org/10.4000/techne.3307

\section{(c) (i) (9)}

La revue Technè. La science au service de l'histoire de l'art et de la préservation des biens culturels est mise à disposition selon les termes de la Licence Creative Commons Attribution - Pas d'Utilisation Commerciale - Pas de Modification 4.0 International. 
Résumé. Souvent, on s'est intéressé aux objets recensés dans les inventaires de Délos en suivant une perspective synchronique et en les considérant comme des éléments figés par leur dédicace à une divinité : le fait que ces objets soient apportés par les pèlerins qui venaient prier les dieux de l'र̂le, puis qu'ils soient consignés sur des stèles de marbre, semblait leur conférer de facto une dimension sacrée. Cette invariabilité supposée des offrandes ne doit cependant pas masquer le caractère bien tangible des artefacts qui, au gré des manipulations à l'intérieur des temples, mais aussi en raison du ravage des années, voient leur aspect originel évoluer dans le sens d'une dégradation naturelle. Cette altération matérielle contredit le caractère sacré inaltérable des dédicaces. Plutôt que d'envisager une fois encore les offrandes du temps de leur splendeur, c'est à partir de leur vieillissement qu'on étudiera leur destin face à l'usure inéluctable: on verra ainsi que tous les objets enregistrés dans les catalogues ne subissent pas le même traitement et que les administrateurs sacrés doivent affronter des problèmes de gestion bien concrets de la désuétude des offrandes.

Mots-clés. Offrande, artefact, inventaire, Délos, vieillissement.
Abstract. Interest has often focused synchronically on objects listed in Delos inventories, regarding them as elements determined by their dedication to a deity: the fact that these objects were brought by pilgrims who came to pray to the island's gods, and were then placed on marble stelae, seemed to confer a sacred dimension upon them. This supposed invariability of offerings should not, however, conceal the tangible character of artefacts which, as a result of the ravages of time and their being handled inside the temples, witnessed the deterioration of their original appearance. These material changes contradicted the inalterable character of the oblations. Rather than once again considering these offerings at the time of their splendour, we shall study their fate as they progressively aged and suffered from inevitable wear and tear: we shall thus see that all the objects recorded in catalogues did not undergo the same treatment and that religious officials had to cope with managerial problems of a truly concrete nature relevant to the desuetude of offerings.

Keywords. Offering, artefact, inventory, Delos, ageing.
À la fin du XIX ${ }^{\mathrm{e}}$ siècle, l'épigraphie délienne se résumait à quelques dédicaces relevées çà et là par des voyageurs. Au cours des grandes fouilles menées à partir des années 1870, plusieurs centaines d'inscriptions furent mises au jour. Parmi ces inscriptions, une grande quantité de décrets permit alors de confirmer des événements qu'on ne connaissait jusqu'alors que par les sources littéraires.

Mais ces textes historiques ne constituent qu'une partie du corpus épigraphique délien, lequel doit son caractère exceptionnel aux quelque cinq cents comptes et inventaires répertoriés et aujourd'hui tous édités. De l'époque classique à l'époque hellénistique, les administrateurs athéniens ou déliens eurent en effet pour tâches:

- de noter chaque année sur une face (fig. 1) de grandes stèles opisthographes ${ }^{1}$ les comptes courants de la gestion du sanctuaire,

- de recenser sur l'autre face le mobilier et les objets nécessaires à l'entretien des temples et à la pratique des cultes,

- d'y consigner surtout les offrandes déposées par ceux qui venaient prier dans les différents sanctuaires de l'île.
Dans chaque inventaire, les descriptions des objets sont regroupées selon deux critères:

- critère topographique, lorsque les administrateurs passent en revue un par un les sanctuaires, puis précisent, à l'intérieur de ceux-là, l'endroit exact de rangement des artefacts, - critère typologique, puisque tout est rassemblé par lots de même taille, de même matière ou surtout de même type. On dégage ainsi quelques grandes catégories (vases, bijoux, armes, etc.).

Le catalogage annuel des offrandes et de tous les objets conservés dans tous les temples et entrepôts était un travail régulier qui occupait une grande partie du temps des administrateurs chargés de cette tâche ${ }^{2}$ : en outre, chaque collège effectuait ce récolement à deux reprises, une fois quand il recevait les offrandes et une seconde fois quand il les transmettait à ses successeurs. Chaque inventaire (et chaque procès-verbal dans les comptes) était donc à la fois une reconnaissance de prise en charge par le collège entrant et un constat de décharge pour le collège sortant. Les administrateurs devaient s'acquitter de cet inventaire en collaboration 


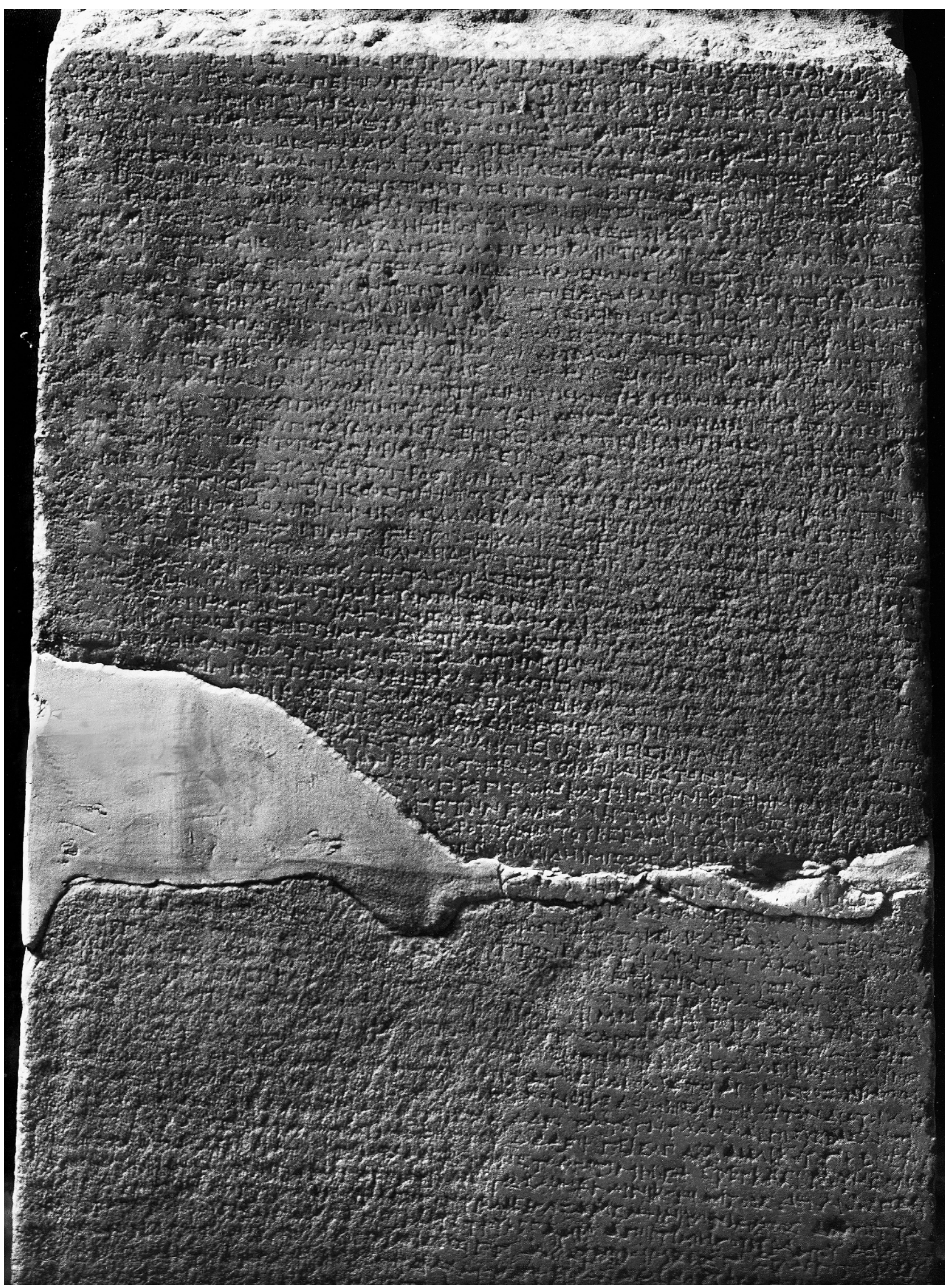

Stèle opisthographe, Inventaire délien $I G$ XI2, 144, face B. ( ) EFA/Philippe Collet. 
avec les prêtres et les prêtresses des lieux de culte quand il y en avait, voire avec le néocore ${ }^{3}$.

L'étude des inventaires dans une perspective diachronique montre que le destin des objets recensés n'est pas synonyme de constance.

Il est d'ailleurs significatif que, pendant longtemps, le terme désignant ces sources épigraphiques d'un type particulier ait été «archives de l'intendance sacrée», englobant dans un amalgame fâcheux tous les artefacts enregistrés ensemble. Encore aujourd'hui, il n'est pas rare de lire l'équation simple: "C'est un objet déposé dans un sanctuaire, c'est donc une offrande ${ }^{4}$ ", en lui conférant de facto un caractère immuable et intangible.

De nombreuses fouilles récentes, notamment dans le monde grec, ont montré que les contextes votifs ne sont pas si évidents à déterminer et qu'on doit se garder de toute assimilation trop péremptoire. Cette nuance concernant l'étude des objets trouvés dans les sanctuaires doit également s'appliquer aux inscriptions qui les mentionnent, et une lecture quasi cursive des inventaires de Délos notamment, permet de reposer la délicate question du statut de l'objet ${ }^{5}$ dont la sacralité n'est pas systématique. Et ce caractère sacré, même quand il est clairement déterminé, n'empêche pas la matérialité d'une offrande qui demeure le résultat d'une production artisanale, qu'elle soit de masse pour les dons stéréotypés, ou individualisée pour des dédicaces plus personnelles. Paradoxalement donc, la vertu sacrée inaltérable d'un objet dédié à une divinité est contrée par toute une série de processus physiques, volontaires lorsqu'il s'agit des manipulations de rangements des prêtres, involontaires lorsque c'est le temps qui fait son œuvre sur des dons entreposés parfois depuis plusieurs décennies.

Pour aider à la description - et donc à l'identification individuelle -, les gestionnaires sacrés avaient à leur disposition de nombreux stratagèmes lexicaux et syntaxiques qui permettaient de coller au plus près de la matérialité du mobilier enregistré ${ }^{6}$. Ainsi, d'une année sur l'autre, le collège en charge de la gestion pouvait rajouter à la liste les nouveaux objets arrivés - offrandes, instruments de culte ou simple mobilier utilitaire -, et contrôler que les précédents enregistrements se trouvaient à la même place.

Si on a pu observer parfois une certaine paresse chez les administrateurs sacrés quand ils recopiaient des inventaires de l'année précédente (ils reproduisaient parfois les fautes de leurs prédécesseurs), il y a cependant une action dont ils s'acquittent parfaitement et qui constitue la raison d'être de ces catalogues: la vérification annuelle systématique de l'état des objets, de leur détérioration, voire de leur perte. Il arrive en effet que les objets s'abîment sous l'effet de facteurs humains ou naturels: si la protection contre le vol et les intempéries était assurée par les édifices où se trouvaient rangées les offrandes, en revanche, le maniement lors des inventoriages annuels, mais également le climat marin propre à Délos pouvaient accélérer le processus d'altération du mobilier et la décomposition des métaux qui entraient dans la fabrication des phiales, des couronnes, des bijoux, pour ne citer que les offrandes les plus fréquentes. Il était donc important pour le bon fonctionnement du sanctuaire, mais aussi pour prouver la probité des administrateurs que ces derniers mentionnent tout type de modification apportée à un objet.

Ce scrupule dans l'enregistrement des détériorations entraîne d'ailleurs une rupture stylistique entre les inventaires de la première domination athénienne au $\mathrm{IV}^{\mathrm{e}}$ siècle et les inventaires de l'Indépendance délienne (314-166 av. J.-C.) : les premiers étaient rigides et calqués sur le modèle attique, tandis que les Déliens introduisent une variété lexicale contrenature, puisqu'elle va à l'encontre de toutes les règles de rigueur de rédaction d'un catalogue. On ne compte pas moins de quinze qualificatifs différents pour exprimer les dommages repérés sur les offrandes enregistrées: si on tente de les traduire en français par des termes variés, on a ainsi : «rongé, dévoré, mangé, délié, détruit, disloqué, fissuré, écrasé, écrasé en morceaux, fêlé, brisé, rayé, fracassé, tronqué, mutilé.» Cette originalité sémantique nous permet aujourd'hui de suivre le parcours d'un objet dans les inscriptions, et de pouvoir reconstituer ce que j'appelle «la vie d'une offrande » : on s'est souvent préoccupé de l'entrée des offrandes dans le sanctuaire, de leur mise en valeur, de leur exposition dans un endroit stratégique du temple, de leur conservation, mais plus rarement de leur pérennité, de leur relégation sur une étagère ou dans une boîte, voire de leur disparition complète. C'est donc «la fin» des offrandes qui nous intéressera ici et surtout le sort qui leur est réservé quand elles ne sont plus mises en avant dans le sanctuaire; on tentera de comprendre si la dégradation est synonyme de désacralisation ou s'il peut y avoir des moyens de maintenir le caractère sacré en dépit d'une déchéance matérielle.

Devant la masse d'objets dégradés ainsi recensés, les prêtres et les administrateurs du sanctuaire ont à leur disposition quatre types d'actions:

I. La réparation;

II. Le recyclage (refonte);

III. Le remplacement (rachat);

IV. L'évacuation dans des lieux de dépôt hors des temples. Cette dernière action pose évidemment la question de la pérennité de la vertu sacrée des objets.

\section{Altération et réparation}

Avant d'envisager la refonte ou le remplacement d'une offrande, on tente de conserver l'objet dans l'état où on le trouve lors de l'inventoriage annuel ${ }^{7}$. Aussi, les termes pour désigner les fragments qu'on rassemble dans des boîtes ou des vases sont-ils variés et sans doute doit-on - comme c'est toujours le cas dans les inventaires déliens - déceler dans ces variations la volonté de distinguer les différents types de débris enregistrés. 
Les $\xi u ́ \sigma \mu \alpha \tau \alpha$ sont les ulcérations faites à une peau grattée et en ce qui concerne un matériau, désignent les petits éléments qui se détachent d'une surface; pour du bois, il s'agit de copeaux, pour des éléments de métal, on peut traduire le terme par «éclats» : à Délos, on recense «des éclats de toutes sortes, provenant des objets en argent du prytanée », á $\lambda \lambda \alpha$

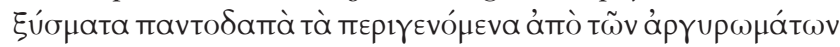

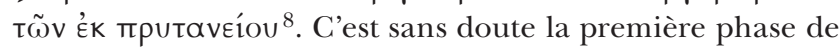
dégradation d'un objet.

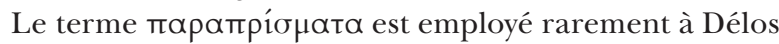
et désigne uniquement les petits éléments qui se détachent des matières naturelles que sont l'ivoire et l'ébène. Ainsi,

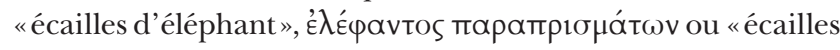
d'ébène ${ }^{9}$ ».

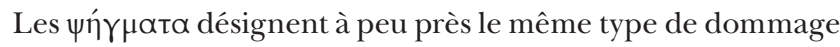
superficiel causé à un objet de métal dans les inventaires déliens. Souvent associés à la formule «et des éléments de

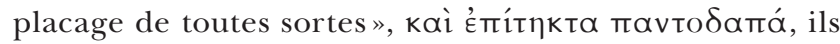
évoquent une pellicule due à une éraflure; ils peuvent provenir de plusieurs types d'objets et on note par exemple des «pel-

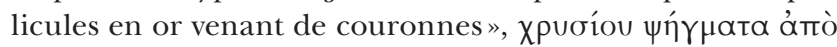

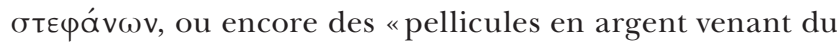

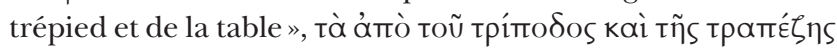

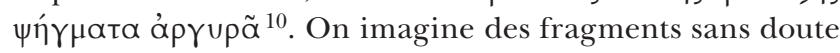
plus gros que les $\xi u ́ \sigma \mu \alpha$ ta précédemment cités dans la mesure où les $\psi \eta ́ \gamma \mu \alpha \tau \alpha$ sont toujours pesés.

Ils sont concurrencés dans les inventaires par un mot

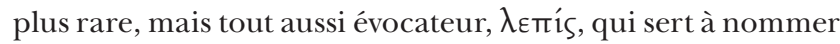
au singulier la couche de placage de certains vases, «brûleparfum en bronze avec une couche d'argent, abîmé ",

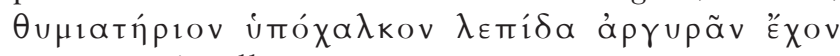

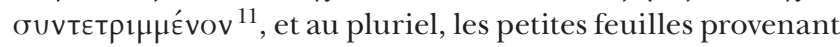
des éraillures du métal, «feuilles de bronze venant des trépieds

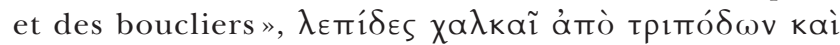
ó $\sigma \pi i \delta \omega v{ }^{12}$.

Enfin, le mot le plus fréquent permettant d'évoquer ce qui reste d'un objet qui s'est cassé est $k \lambda \alpha$ ó $\mu \alpha \tau \alpha$, «les fragments", qui peut s'appliquer aussi bien à des éléments détachés - mais non brisés - d'un artefact, "poignées en fer et autres

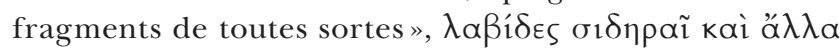

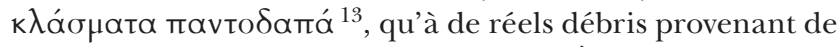
couronnes, de lampes ou de récipients. À la différence des pellicules, les fragments ne sont jamais ${ }^{14}$ pesés mais comptés, et peuvent être sans doute identifiés en raison de leur taille et de leur forme.

Entre la phase de maintien en l'état d'une offrande altérée dans le temple, la phase de collation des éléments détachés des offrandes et la procédure de refonte ou de remplacement, on confie d'abord aux artisans le soin de réparer les objets déposés dans le sanctuaire. On rémunère ainsi «Theutimides

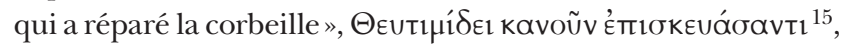

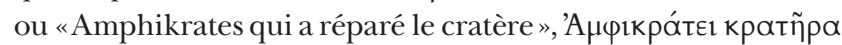

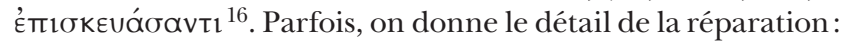
ainsi «Hérakleidès a réparé le cratère et l'anse du cothôn»,

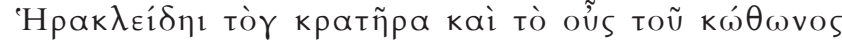

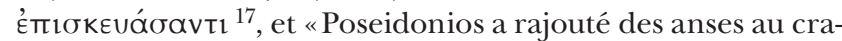

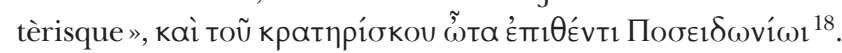

Ces réparations portent sur des objets qui ont pu être dédiés, mais aussi employés dans les différents rituels des sanctuaires déliens et rien dans les descriptions ne permet de déduire clairement leur statut votif. De même, avec la rémunération accordée à «Theophantos qui a réparé le char

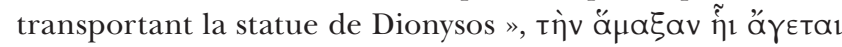

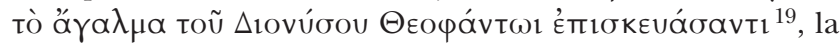
limite entre sacré, rituel et votif est mince: de part son lien avec la statue de culte, le char est sacré; il peut être utilisé lors de la procession accompagnant la statue hors du temple une fois par an et donc se retrouve dans une fonction rituelle. Enfin, se pose l'éternelle question de l'origine des statues de culte - et de leurs accessoires, fabriquées spécialement pour un temple mais qui ont pu faire l'objet d'une dédicace de la part d'une communauté, d'une cité, voire d'un individu. Dans tous ces cas néanmoins, on constate une volonté de maintien des objets sacrés par un entretien régulier qui porte sur différents types d'objets et ruine ainsi toute classification marchande: une offrande n'appartient pas à un système économique et les soins qu'on lui prodigue ne tiennent pas compte de son éventuelle valeur marchande.

\section{Refonte}

La refonte des offrandes métalliques est en général accompagnée d'un processus administratif assez complexe qui semble avoir un double but: éviter au maximum la possibilité de tricherie et de fraude lorsque le métal est précieux ${ }^{20}$ et surtout, prouver qu'une offrande reste une offrande, quelle que soit sa forme de départ et d'arrivée.

À Athènes, quelques rares décrets mentionnent indirectement les procédures. $I G \mathrm{II}^{2}, 333$, évoque le regroupement des offrandes de même métal sanctuaire par sanctuaire, dans la perspective de la refonte. Le compte $I G \mathrm{II}^{2}, 1496$, signale les rémunérations d'un orfèvre chargé de l'åmókavoıs, action de faire chauffer («brûler») ensemble les métaux, livre le calcul de la perte d'or observée lors de la fonte, puis donne le poids des grains d'or récupérés dans les cendres une fois l'opération achevée.

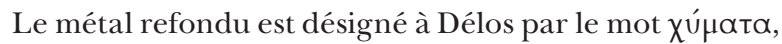
littéralement «les liquides», c'est-à-dire les métaux refondus et moulés en lingots. Il est précisé dans les comptes à partir de quels objets sont fabriqués les $\chi u ́ \mu \alpha \tau \alpha$ : de la refonte d'une

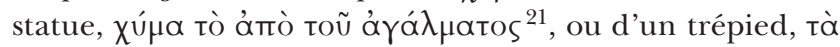

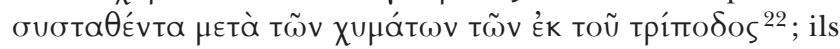
sont le plus souvent en or et donc rangés dans des boîtes avec les fragments du même métal non refondus, $\chi \rho \rho^{\prime}$ íov $^{\prime} \gamma$

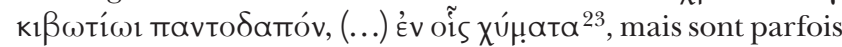

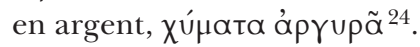

Les lingots sont transmis et enregistrés dans les inventaires et non dans les comptes, ce qui leur ôte toute valeur marchande et les conforte dans le système votif puisqu'ils sont uniquement 
issus des offrandes refondues: "À partir des pellicules d'or et des couronnes qui se sont disloquées, et que nous avons transmises aux artisans choisis par décret par le demos pour effectuer la refonte, voici les lingots en or que nous avons

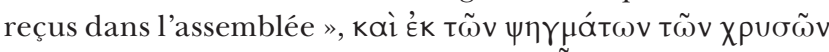

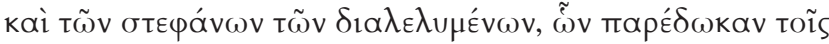

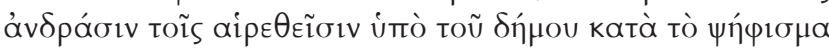
 $\chi \rho \cup \sigma \tilde{\alpha}^{25}$; suit une énumération du premier lingot avec son poids, du deuxième, etc.

Parfois, le résultat de la refonte n'est pas un lingot, et on le désigne alors simplement par le métal: «Et à partir des

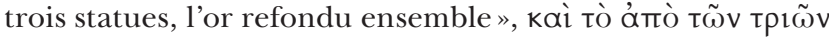

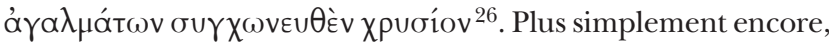
on rassemble les fragments d'or ou d'argent sans les refondre

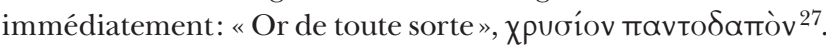

Plus rares sont les mentions de nouveaux objets fabriqués à partir de la refonte. On rémunère ainsi un artisan qui a

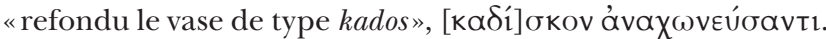
Le texte lacunaire interdit de restituer l'objet refondu avec

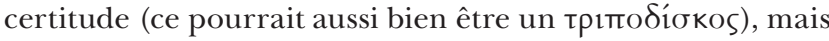
le préverbe ơva- signale sans aucun doute qu'il ne s'agit pas d'une fabrication nouvelle, mais d'un recyclage à partir d'offrandes en métal plus anciennes.

Lors de la seconde domination athénienne, les administrateurs sacrés mentionnent une corbeille en argent «que nous avons fabriquée suivant la décision de l'épimélète", et se sentent obligés de préciser dans une description complètement anormale pour un catalogue d'offrandes: "Cette corbeille, nous l'avons fabriquée à partir des plaquettes votives détruites et inutiles, des torches dans le sanctuaire qui étaient déjà là avant l'année de notre administration. Parmi ces torches et ces fragments métalliques, il y a tous ceux qui ont été refondus comme cela est mentionné dans le texte de la

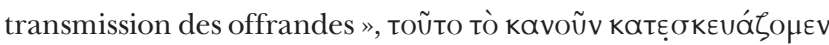

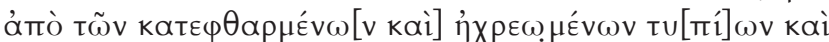

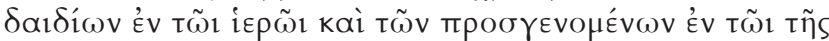

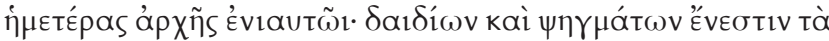

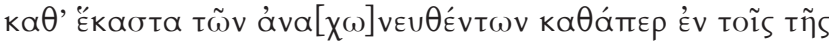

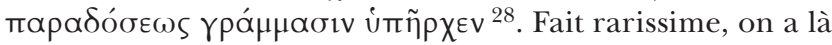
une double refonte: des torches et des fragments métalliques divers avaient servi à fabriquer d'autres torches, ainsi que des plaquettes votives lesquelles, par la suite, ont été détruites,

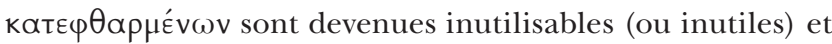
ont donc été une nouvelle fois refondues en corbeille. C'est là un exemple original de recyclage perpétuel des offrandes.

\section{Le remplacement par rachat}

Dans le premier cas comme dans le deuxième, l'offrande demeure dans le sanctuaire et conserve une fonction sacrée inaliénable à partir du moment où elle a été déposée à la divinité. Le remplacement d'un don par un autre est donc la solution la plus commune en cas de dégradation et les formules des inventaires déliens sont courantes: «À la place de la phiale thérikléios qui a été mise au rebut, offerte par Pythéios, une

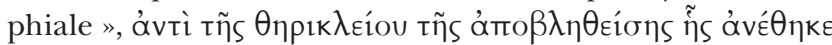
Пú$\theta \varepsilon 10 \varsigma, ~ \varphi ı \alpha ́ \lambda \eta^{29}$, « rhodiakon à la place de la phiale chélidonéios

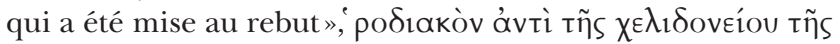

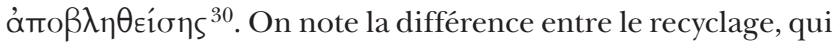
se traduit par la conservation de la matière initiale des offrandes grâce à la refonte, et le remplacement par un objet qui n'est pas forcément identique, et sur lequel il y aura eu transfert du caractère sacré: on n'hésite pas à mettre au rebut, mais en permettant aussitôt à un autre objet d'acquérir cette sacralisation. L'usage du participe aoriste du verbe ómoßá $\lambda \lambda \omega$ pour désigner l'évacuation de certaines dédicaces est propre aux inventaires déliens. On emploie le même verbe lorsqu'on pèse les offrandes: «Cela a été pesé sous le même [archonte] et on a trouvé, avec les objets mis au rebut, un poids de 2 drachmes ",

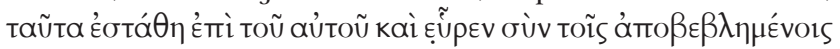
$\delta \rho \alpha \chi$. - r. La manière de mettre au rebut n'est pas indiquée dans les inventaires. Les recherches archéologiques menées depuis longtemps à Délos permettent néanmoins de renoncer à l'hypothèse de favissae et d'enfouissements rituels, caractéristiques d'autres lieux de culte ${ }^{31}$. Les oikoi évoqués ci-dessous constituent une des réponses possibles à cette question.

Le déplacement du sacré d'un objet à l'autre s'effectue de manière plus obvie encore quand les administrateurs remplacent un animal dédié par un objet pérenne: «Vase hedupotis à la place des chevreaux offerts par Hegemon »,

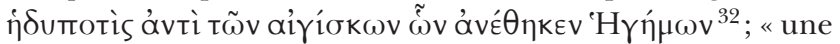
sorte de kylix à la place du bélier offert par Demetrios",

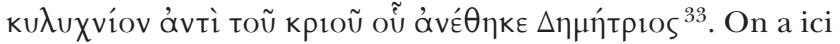
l'exemple d'une superposition de strates dans les fonctions à attribuer à l'animal dans un sanctuaire: un bélier pouvait être offert sans être destiné au sacrifice pour autant, et l'équivalence animal-rituel doit alors être nuancée; bien qu'offert, il pouvait cependant être vendu et on voit s'introduire une action commerciale dans les pratiques votives, puisque du prix de la vente dépend l'artefact substitut. Ainsi, la dédicace, le sacrifice et la vente d'une offrande animale sont autant d'actes où le processus de sacralisation ou de ritualisation est maintenu.

\section{L'évacuation dans des lieux de dépôt hors des temples}

La quatrième solution adoptée par les administrateurs est l'évacuation d'une offrande hors d'un temple: le transfert des objets sacrés dans les oikoi est un phénomène courant à Délos, mais les motivations sont peu clairement affirmées dans les inventaires: on a longtemps supposé que c'était le manque de place qui justifiait cette manutention et que lors de l'arrivée de nouvelles offrandes, on déplaçait les anciennes, selon des critères qui restent encore à déterminer. L'état de dégradation des objets peut constituer une autre raison à ces déplacements puisque les temples se devaient d'être une sorte de vitrine prestigieuse des dons reçus par les divinités. 
Cette pratique délienne s'observe également à Athènes mais, a contrario, on trouve, à Rhodes par exemple, des règlements interdisant l'exportation et la destruction des offrandes: «Il est défendu de sortir les ex-voto du temple et de les

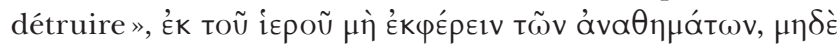

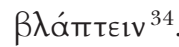

À Délos, ce phénomène de transfert existait certes d'un temple à un autre, comme dans le cas des offrandes qui viennent du Sarapieion et se retrouvent dans l'Artémision :

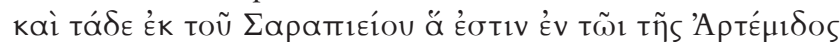
$v \alpha \tilde{\omega} 1^{35}$. Mais on demeurait là dans le domaine géographique et symbolique du sacré.

Le cas est un peu différent pour les offrandes rangées dans les oikoi déliens, construits aux frais de certaines cités (Naxos, Andros, Carystos) et apparentés à des entrepôts où on laisse surtout le bois, les tuiles et les outils destinés aux réparations dans le sanctuaire. Leur rôle est bien différent des oikoi de Delphes, qu'on appelle les trésors, et qui sont eux-mêmes des offrandes censées pour la plupart représenter la dîme d'une aubaine, victoire militaire ou prospérité économique.

Le Porinos naos à Délos, littéralement «le temple de Poros », sert juste au final d'entrepôt à offrandes, et dans les inscriptions du $\mathrm{II}^{\mathrm{e}}$ siècle av. J.-C., il est renommé Porinos oikos sans plus aucune valeur sacrée.

Le mot oikos à Délos désigne donc systématiquement les locaux qui servent de lieu de stockage de matériaux de construction et qui n'abritent pas de statues cultuelles, mais bien souvent des offrandes rangées par types sur des étagères. Ainsi, dès les années 340-335, à côté des poutres de chêne, l'oikos des Naxiens abrite des récipients de bronze (marmite,

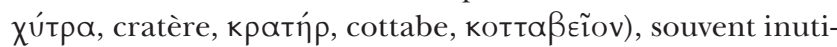

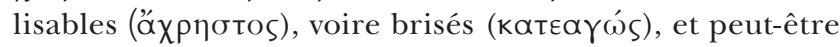
est-ce la raison pour laquelle ils se transforment eux-mêmes en boîtes de rangement destinés à des fragments de bronze

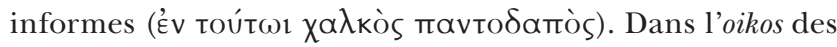
Andriens, à l'Indépendance, on recense de l'or provenant

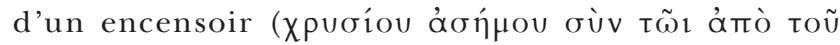
$\theta$ uнı⿻т丨íou), et on y place des bagues qui se trouvaient auparavant dans un tronc à offrandes de la Nesos ${ }^{36}$, comme beaucoup d'objets en argent, dont une sorte de phiale remplaçant la therikleios offerte par Demoson et mise au rebut,

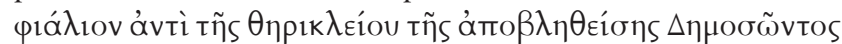

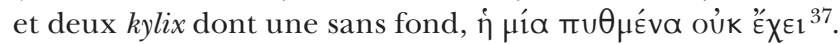
Les raisons du dépôt de ces offrandes sont moins évidentes que pour l'oikos des Naxiens, car toutes ne sont pas abîmées.

La question de l'interprétation de ces édifices reste ouverte selon qu'on se range à un avis ancien qui en fait les prémices des temples déliens, à un avis datant d'il y a trente ans, qui fait de ces édifices des lieux sacrés, mais en aucun cas des temples, ou bien à un avis plus récent qui propose de refuser la traduction d'oikos par «trésor», mais d'y substituer les mots «foyer, amicale», perdant ainsi complètement la notion de consécration religieuse propre à ces bâtiments. Les objets qui y sont stockés ne permettent pas de préciser le statut de ces oikoi; doit-on penser que ce sont des édifices religieux au seul prétexte qu'ils abritent des offrandes ou qu'ils sont administrés par des néocores ${ }^{38}$ ? Le maintien du caractère sacré des objets entreposés dans des lieux autres que les temples est encore plus délicat à envisager quand on sait que les inventaires enregistrent aussi des offrandes en argent dans le prytanée et il faudrait suivre les dons sur plusieurs décennies d'inventaires pour observer la mobilité de ces objets avant de comprendre s'ils demeurent sacrés ou pas.

Quelle que soit la valeur des objets rangés dans ces entrepôts, on peut néanmoins remarquer que leur seule place dans des lieux autres que les temples élimine d'emblée l'idée d'un rituel accompagnant leur entrée. Les donateurs ne jouent aucun rôle dans le dépôt de leur offrande et il n'est même pas certain que le personnel chargé de la réception des dons avait une place dans l'élaboration d'un rite autour de ceux- $\mathrm{ci}^{39}$. L'offrande se trouvant dans un entrepôt dès son arrivée perd donc toute possibilité d'être assimilée à un objet ritualisé par une action particulière au moment de sa dédicace, mais cela lui ôte-t-il pour autant son caractère sacré?

Les manières de décrire l'usure des objets et les moyens mis en œuvre afin de les conserver, les réparer, les remplacer ou les évacuer sont nombreux dans les inventaires déliens. Les décisions prises pour l'application d'un type de traitement plutôt que d'un autre ne dépendent pas de l'objet lui-même, mais de son état. Cela pose le principe d'égalité de la valeur sacrée des offrandes qui confirme l'absence d'une hiérarchisation commerciale. Au-delà de la volonté publicitaire habituelle des inventaires déliens, le but de ces soins apportés à tous les dons témoigne du perpétuel souci des administrateurs sacrés de se protéger des accusations de mauvaise gestion et de témoigner, par la variété du lexique, de la vivacité des pratiques votives de l'île.

\section{Notes}

Liste des sigles et abréviations de collections

ID: Corpus des Inscriptions de Délos, Paris. $I G$ : Inscriptiones Graecae, Berlin.

1. Les stèles de marbre de Délos étaient gravées sur les deux faces.

2. Les administrateurs sacrés étaient des Athéniens à l'époque où ces derniers dominaient Délos, puis lors de l'Indépendance de l'île (314-166 av. J.-C.), les administrateurs furent choisis parmi les Déliens et se prénommèrent hiéropes. 3. Personnel chargé de l'entretien courant des temples.

4. Rey-Vodoz, 1991, p. 215, en introduction, écrit: «Le fait de se situer dans un espace dont le caractère sacré ne fait aucun doute (...) permet de se dispenser d'une première discussion sur l'interprétation cultuelle du contexte. » Elle nuance toutefois son propos ensuite par «l'un des problèmes fondamentaux est de différencier de façon sûre un matériel votif d'un matériel résiduel propre à tout vestige."

5. Prêtre, 2014 (sous presse).

6. Prêtre, 2012, p. 20-38.

7. Prêtre, 1999, p. 389-396.

8. ID 442, 1. 86 .

9. IG XI 2, 298A, 1. 181. ID 320B, 1. 68

10. ID 396B, 1. 80 et ID 442B, 1. 89. La 
restitution dans l'editio princeps d'un

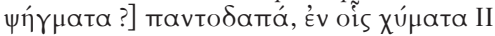
doit donc être supprimée car il est impossible de recenser des lingots parmi des écailles.

11. $I G$ XI 2, 161B, 1. 11.

12. $I G$ XI $2,199 \mathrm{~B}, 1.86$.

13. ID 372B, 1. 36.

14. On a aussi parfois la tentative de rassemblement des différents éléments cassés d'une offrande: "cyathe brisé et

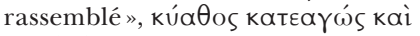

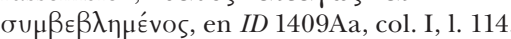

15. IG XI 2, 219A, 1. 39.

16. $I G$ XI 2, 147A, 1. 6.

17. $I G \mathrm{XI} 2,154 \mathrm{~A}, 1.34$

18. IG XI 2, 203A, 1.35

19. IG XI 2, 161 A, 1. 91 .

20. Woodward, 1951, p. 109-111.

21. ID $385 \mathrm{~A}, 1.7$.

22. ID 399B, 1. 28

23. ID $385 \mathrm{~A}, 1.6$

24. ID $439,1.86$

25. ID 442B, 1. 120.

26. ID $313,1.45$.

27. IG XI 2, 203B, 1. 43 .
28. ID 1442B, 1. 64

29. IG XI 2, 161B, 1. 64.

30. ID 298A, 1. 109

31. Voir Patera, 2012, p. 193-194.

32. ID 298A, 1. 110

33. $I G$ XI 2, 224B, 1. 26.

34. $L S A M \mathrm{n}^{\circ} 74$.

35. ID 1403Bb, col. II, 1. 40.

36. Tréheux, 1995, p. 187-209.

37. $I G$ XI 2, 287B, 1. 89.

38. IG XI 2, 287A, 1. 78, mentionne le néocore de l'oikos des Carystiens.

39. Voir Pirenne-Delforge, 2010, p. $121-141$

\section{Bibliographie}

Patera I., 2012, Offrir en Grèce ancienne. Gestes et contextes, Franz Steiner Verlag, Stuttgart.

Pirenne-Delforge V., 2010, "Greek Priests and 'Cult-Statues': In How Far are they Unnecessary?", dans Mylonopoulos J. (éd.), 2010, Divine Images and Human Imaginations in Ancient Greece and Rome, Brill, Leyde, p. 121-141.

Prêtre C., 1999, «Le matériel votif à Délos. Exposition et conservation ", $\mathrm{BCH}$, p. 389-396.

Prêtre C., 2012, Kosmos et kosmema. Les offrandes de parure dans les inscriptions de Délos, Kernos, Liège.

Prêtre C., 2014, «L'offrande dans les inventaires de Délos: objet rituel ou objet sacré? ", RHR (sous presse).

Rey-Vodoz V., 1991, «Les offrandes dans les sanctuaires gallo-romains", dans Brunaux J.-L. (éd.), 1991, Les sanctuaires celtiques et leurs rapports avec le monde méditerranéen, Errance, Paris, p. 215 220 .

Tréheux J., 1995, «Archéologie délienne: l'Artémision 'Ev Níowı localisation et histoire », Journal des Savants, p. 187-

Woodward A. M., 1951, «'Aпоvбía», Numismatic Chronicle, p. 109-111. 